hep-ph/0302056

CALT-68-2427

March 17, 2003

\title{
Softening the Naturalness Problem
}

\author{
Xavier Calmet ${ }^{1}$ \\ California Institute of Technology, Pasadena, California 91125, USA
}

\begin{abstract}
It was observed by Veltman a long time ago that a special value for the Higgs boson mass could lead to a cancellation of the quadratically divergent corrections to the Higgs boson's squared mass which appear at one loop. We present a class of low energy models that allow to soften the naturalness problem in the sense that there can be a cancellation of radiative corrections appearing at one loop. The naturalness problem is shifted from the one $\mathrm{TeV}$ region to the $10 \mathrm{TeV}$ region. Depending on the specific model under consideration, this scale can even be shifted to a higher energy scale. Signatures of these models are discussed.
\end{abstract}

\footnotetext{
${ }^{1}$ email:calmet@theory.caltech.edu
} 


\section{Introduction}

The standard model is a gauge theory based on the structure group $\mathrm{SU}(3)$ $\times \mathrm{SU}(2) \times \mathrm{U}(1)$ [1,2]. The electroweak gauge symmetry is spontaneously broken by means of the Higgs mechanism [3. This mechanism requires the inclusion in the model of a fundamental scalar field which is charged under $\mathrm{U}(1)$ and is in the fundamental representation of $\mathrm{SU}(2)$. It is often argued that the standard model with the Higgs mechanism as a mechanism for gauge symmetry breaking cannot be a theory valid over a wide range of energies because the squared mass of a scalar field receives corrections that are quadratic divergent if a naive cutoff is used to regularize the model. In the standard model one obtains

$$
m_{H}^{2} \approx m_{H}^{0}{ }^{2}+\frac{3 g^{2} \Lambda^{2}}{32 \pi^{2} m_{W}^{2}}\left(m_{H}^{2}+2 m_{W}^{2}+m_{Z}^{2}-4 \sum_{f}\left(\frac{n_{f}}{3}\right) m_{f}^{2}\right)
$$

in the one loop approximation [4]. The mass term of the Higgs boson is denoted by $m_{H}$, that of the W-boson by $m_{W}$, that of the Z-boson by $m_{Z}$ and that of the top-quark is denoted by $m_{t}$. Finally $n_{f}$ is the number of flavors propagating in the loop and $g$ is the $\mathrm{SU}(2)$ gauge coupling constant. If the cutoff is large, e.g. of the order of a possible grand unification scale $10^{16} \mathrm{GeV}$, it requires an unnatural adjustment between the bare mass $m_{H}^{0}$ of the scalar field and the "corrections" to insure a physical Higgs mass in the $100 \mathrm{GeV}$ region [5]. This is known as the naturalness problem. It should nevertheless be noted that the standard model is renormalizable 66. Quadratic divergences can be absorbed in the parameters of the standard model in a way which is mathematically completely consistent.

An obvious solution to the naturalness problem is to avoid the Higgs mechanism and the inclusion of fundamental scalars in the model like in e.g. technicolor theories or top condensate models [7]. Another approach is to embed the standard model into another more fundamental theory where quadratic divergences are either absent like in supersymmetric models [8] or small like in models with extra-dimensions [9] because in that case the fundamental scale of nature is assumed to be not much larger than the electroweak scale.

Recently another point of view has been revived. It had been proposed a long time ago [10], that the Higgs boson could be a pseudo-Goldstone boson. This idea has recently been revived in the form of the Little Higgs models 
[1]. The Little Higgs models have been shown to possess an approximate symmetry that can protect the Higgs mass from radiative corrections if the cutoff is not too large i.e. $10 \mathrm{TeV}$. Unfortunately the simplest examples of that class of models do not automatically have a custodial symmetry and are thus potentially severely constrained by experiments [12].

Long before that, it had been speculated that a special value for the Higgs boson mass could cancel the quadratic divergences corrections to the Higgs boson mass [4. This leads to the so-called Veltman's relation:

$$
m_{H}^{2}=4 m_{t}^{2}-2 m_{W}^{2}-m_{Z}^{2}
$$

But, even if this relation was fulfilled, i.e. if the Higgs boson mass was of the order of $316 \mathrm{GeV}$, it does not hold beyond the one loop approximation. The consequences of a possible cancellation of the logarithmic divergent terms have been considered in [13. Note that there is no need to require an exact cancellation of the one loop quadratic divergences [14.

In this work we shall argue that the observation made by Veltman some 22 years ago can be revived in a modern framework. We shall present a class of models for which, as in the Little Higgs models case, we do not describe the high energy completion and we shall thus assume that our models have a cutoff in the $10 \mathrm{TeV}$ region. The simplest way to do that is to assume that the standard model Higgs boson mass is of the order of $316 \mathrm{GeV}$ and that this model has a cutoff of $10 \mathrm{TeV}$. We note that such a high mass for the Higgs boson seems to be in contradiction with fits based on electroweak precision measurements [15. Furthermore, in that case, there is no way to push the cutoff scale above the $10 \mathrm{TeV}$ scale. Our basic observation is that if there is some new physics beyond the standard model with a new bosonic degree of freedom $\phi$ that is coupled to the standard model in a minimal way i.e.

$$
\alpha h^{\dagger} h \phi^{\dagger} \phi
$$

where $h$ is the standard model Higgs boson and $\alpha$ is a parameter of order one, then the Higgs boson mass can naturally be of the order of $100 \mathrm{GeV}$ if the models have a cutoff of the order of $10 \mathrm{TeV}$. Furthermore, depending on the model under consideration, this cutoff scale can be pushed upwards. The naturalness problem is not solved by these models, but is only soften. It must be emphasized that these models are theoretically not as compelling as the Little Higgs models, because they do not have an approximate symmetry 
that protects the Higgs boson squared mass against radiative corrections but they have a custodial symmetry and are thus not constrained by experiments. These models are semi-natural in sense proposed by Veltman 4. A new boson that couples to the Higgs boson of the standard model according to (3) implies a correction to the Higgs boson squared mass given by:

$$
\begin{aligned}
m_{H}^{2} \approx & m_{H}^{0^{2}}+\alpha \Lambda_{c}^{2} \\
& +\frac{3 g^{2} \Lambda^{2}}{32 \pi^{2} m_{W}^{2}}\left(4 v^{2} \lambda+2 m_{W}^{2}+m_{Z}^{2}-4 \sum_{f}\left(\frac{n_{f}}{3}\right) m_{f}^{2}\right)+\frac{\Lambda^{2}}{4 \pi^{2}} \alpha,
\end{aligned}
$$

where $\lambda$ is the Higgs boson self-coupling, $v=174 \mathrm{GeV}$ the vacuum expectation value and $\Lambda_{c}$ is the scale associated with the new physics beyond the standard model. Note that depending on the model our definition of $\Lambda_{c}$ includes a potential mixing angle between the scales of the different scalar sectors. In the sequel we shall describe two classes of models that should be considered as low energy effective theories of an unknown high energy theory. If we introduce the operator (3) in the standard model, we potentially introduce a new naturalness problem. Because $\phi$ is a bosonic degree of freedom, its squared mass will in general receive quadratic corrections. We have identified two classes of models where this problem is under control. The first class of models are models where $\Lambda_{c}$ is identical to the standard model electroweak scale, and where a symmetry implies that the corrections to the squared mass of the scalar field $\phi$ are identical to those of the scalar fields $h$. The second class of models are models where the scale involving the second scalar field $\phi$, i.e. its mass, is rather near to the cutoff scale $\Lambda$. The corrections to its squared mass are thus small.

Clearly, if $\alpha$ is a positive parameter of the order of one, a cancellation, or partial cancellation, of the the quadratic corrections can take place and the Higgs boson mass can naturally be of the order of $100 \mathrm{GeV}$ if the cutoff is of the order of $10 \mathrm{TeV}$. Note that the the two loop corrections are expected to be of the order of $\left(\frac{1}{16 \pi^{2}}\right)^{2} \Lambda^{2}$ and are thus small if the cutoff is as low as $10 \mathrm{TeV}$. The corrections to the potential of the new scalar degree of freedom are model dependent and shall be discussed below for each model considered. Note also that if $\alpha$ is a negative parameter of order one and if $\Lambda_{c}<\Lambda$, there is a negative contribution at tree level to the Higgs boson mass that can, in principle, reverse the sign of the Higgs boson squared mass and thus trigger the Higgs mechanism in the electroweak sector of the standard model. In that scenario we have to require that the new scale is lower than the cutoff scale to 
be certain that the low energy effective theory remains valid. Nevertheless, in that case, there is, in general, no cancellation of the quadratic divergences.

In the first section, we shall consider a model where $\Lambda_{c}$ is assumed to be equal to the scale of the electroweak interaction. In the second section we will describe a model where mass of the second scalar field is assumed to be near to the cutoff of the models. We then conclude.

\section{$2 \quad$ A standard model replica}

Let us consider a model based on the gauge group $\mathrm{SU}(3)_{\bar{C}} \times \mathrm{SU}(2)_{\bar{L}} \times$ $\mathrm{U}(1)_{\bar{Y}} \times \mathrm{SU}(3)_{C} \times \mathrm{SU}(2)_{L} \times \mathrm{U}(1)_{Y}$, where $\mathrm{SU}(3)_{\bar{C}} \times \mathrm{SU}(2)_{\bar{L}} \times \mathrm{U}(1)_{\bar{Y}}=\mathrm{G}_{n}$ is the gauge group describing the physics beyond the standard model and $\mathrm{SU}(3)_{C} \times \mathrm{SU}(2)_{L} \times \mathrm{U}(1)_{Y}=\mathrm{G}_{S M}$ is the standard model gauge group. Both $\mathrm{SU}(2)$ groups have the usual weak gauge coupling $g$ and both $\mathrm{SU}(3)$ groups have the usual strong coupling $g_{s}$. Furthermore, we assume also that both $\mathrm{U}(1)$ groups have the same usual gauge coupling $g^{\prime}$. The number of fields of the model is thus doubled in comparison to the standard model (see table 11), and there is a discrete symmetry transforming the standard model fields (Higgs boson included) into the fields charged under $\mathrm{G}_{n}$. The motivation to consider this model is that because of the discrete symmetry, the corrections to the masses of both scalar degrees of freedom are identical. Therefore only one relation has to be fulfilled to insure the naturalness of the model if the cutoff is assumed to be around $10 \mathrm{TeV}$. The Lagrangian of the model is given by

$$
\mathcal{L}_{\text {rep }}=\mathcal{L}_{S M}+\mathcal{L}_{n}-\alpha h^{\dagger} h \Phi^{\dagger} \Phi
$$

where $h$ is the $\mathrm{SU}(2)_{L}$ scalar doublet and $\Phi$ is the $\mathrm{SU}(2)_{\bar{L}}$ scalar doublet. This is the most generic, gauge invariant Lagrangian. $\mathcal{L}_{S M}$ is the standard model Lagrangian and $\mathcal{L}_{n}$ is the Lagrangian containing the new fields, it is obtained by applying the discrete symmetry mentioned above on $\mathcal{L}_{S M}$. Note that this model has a custodial symmetry and is thus not in contradiction with electroweak precision measurements. The potential of the model reads:

$$
V(h, \Phi)=m_{s}^{2} h^{\dagger} h+m_{s}^{2} \phi^{\dagger} \phi+\lambda\left(h^{\dagger} h\right)^{2}+\lambda\left(\Phi^{\dagger} \Phi\right)^{2}+\alpha h^{\dagger} h \Phi^{\dagger} \Phi .
$$

The vacuum expectation value of the scalar fields $v$ is given by:

$$
v=\sqrt{\frac{-m_{s}^{2}}{2 \lambda+\alpha}},
$$


note that $m_{s}^{2}<0$, the potential is bounded from below if $2 \lambda>\alpha$. The parameter $m_{s}$ receives the usual quadratic divergencies:

$$
\begin{aligned}
m_{s}^{2} \approx & m_{s}^{02}+\alpha v^{2} \\
& +\frac{3 g^{2} \Lambda^{2}}{32 \pi^{2} m_{W}^{2}}\left(4 v^{2} \lambda+2 m_{W}^{2}+m_{Z}^{2}-4 \sum_{f}\left(\frac{n_{f}}{3}\right) m_{f}^{2}\right)+\frac{\Lambda^{2}}{4 \pi^{2}} \alpha .
\end{aligned}
$$

If we require the complete cancellation of the quadratic divergent corrections induced at one loop, we obtain the analog of Veltman's condition:

$$
\lambda+\frac{1}{3} \alpha=\frac{g^{2}}{8 m_{W}^{2}}\left(4 \sum_{f}\left(\frac{n_{f}}{3}\right) m_{f}^{2}-2 m_{W}^{2}-m_{Z}^{2}\right) \approx 0.86,
$$

which insures the cancellation of the quadratic divergences induced by the one loop corrections.

\section{Signatures}

The operator $h^{\dagger} h \Phi^{\dagger} \Phi$ induces a mixing between the two scalar doublets. Let us denote the mass eigenstates by $h_{1}$ and $h_{2}$. One finds:

$$
\begin{aligned}
& h_{1}=\frac{1}{\sqrt{2}} h^{0}-\frac{1}{\sqrt{2}} \phi^{0} \\
& h_{2}=\frac{1}{\sqrt{2}} h^{0}+\frac{1}{\sqrt{2}} \phi^{0},
\end{aligned}
$$

i.e. the mixing is maximal. After this diagonalization procedure, $h_{1}$ and $h_{2}$ couple to both the fermions and their replicas. There is nevertheless no new source of neutral flavor changing. The replicas are only extremely weakly coupled to the standard model particles, the only possible interaction is mediated by the scalar bosons. In that sense the decays of the scalar bosons to replicas should be considered as missing energy decay modes. Therefore, the observable spectrum of the theory is the standard model with a further neutral scalar boson. The squared masses of the scalar bosons are given by

$$
\begin{aligned}
& m_{h_{1}}^{2}=m_{h}^{0^{2}}-\alpha v^{2}=2(2 \lambda-\alpha) v^{2} \\
& m_{h_{2}}^{2}=m_{h}^{0^{2}}+3 \alpha v^{2}=2(2 \lambda+\alpha) v^{2},
\end{aligned}
$$

with $v=174 \mathrm{GeV}$. Let us assume that the lightest Higgs boson has a mass $m_{h_{1}}$ of about $130 \mathrm{GeV}$ and that the one loop quadratically divergent corrections cancel completely, one finds $m_{h_{2}}=349 \mathrm{GeV}$. Note that the production 
and decay modes of these scalar bosons are quite different from the standard model case. At an electron positron collider the production rate is $1 / 2$ of that of the standard model because of the mixing between the two scalars reduces the coupling of each of the scalars to the $\mathrm{SU}(2)_{L}$ gauge bosons by a factor $1 / \sqrt{2}$. One has schematically $\sigma\left(e^{+} e^{-} \rightarrow H Z^{*}\right) \sim \frac{1}{2} \sigma\left(e^{+} e^{-} \rightarrow h_{1} / h_{2} Z^{*}\right)$ where is it understood that the appropriate scalar mass has to be used in the formula. Similarly the couplings to fermions charged under $\mathrm{G}_{S M}$, i.e. the standard model fermions, is reduced by the same factor. This implies that the LEP limits for these scalar bosons are less stringent in this model. The lightest Higgs boson decays as in the standard model dominantly to $b$-quarks if its mass is around $100 \mathrm{GeV}$. The cross section is $\sigma\left(e^{+} e^{-} \rightarrow b \bar{b} Z^{*}\right)=\left.\frac{1}{4} \sigma\left(e^{+} e^{-} \rightarrow b \bar{b} Z^{*}\right)\right|_{S M}$, and is thus much smaller than in the standard model. We have assumed that only the lightest Higgs boson contributes at a significant level. The cross section to missing energy, i.e. when the Higgs boson decays to the $b$-quark replicas, is equal in magnitude to the $b-\bar{b}$ decay mode: $\sigma\left(e^{+} e^{-} \rightarrow\right.$ missing energy $\left.Z^{*}\right)=\sigma\left(e^{+} e^{-} \rightarrow b \bar{b} Z^{*}\right)$. The missing energy corresponds to a scalar of mass $m_{h_{1}}$. Notice that both scalars couple both to the standard model particles and to the replica particles. This implies that fifty percent of the scalar bosons should be missing energy decays. Note that the production at a hadron collider, where one of the main production mode for the Higgs boson is via a top quark triangle, will also be suppressed by a factor $1 / 2$ compared to the standard model expectation.

\subsection{Elusive new physics}

Let us consider the same model as described above but we shall now assume that the $\mathrm{SU}(2)_{\bar{L}}$ gauge symmetry remains unbroken. The scalar potential is assumed to be:

$$
V(h, \Phi)=m_{s}^{2} h^{\dagger} h-m_{s}^{2} \phi^{\dagger} \phi+\lambda\left(h^{\dagger} h\right)^{2}+\lambda\left(\Phi^{\dagger} \Phi\right)^{2}+\alpha h^{\dagger} h \Phi^{\dagger} \Phi,
$$

the discrete symmetry is softly broken by the mass terms of the scalar fields.

The vacuum expectation values are given by $v_{h}=\sqrt{\frac{-m_{s}^{2}}{2 \lambda+\alpha}}$ and $v_{\Phi}=0$. This implies that there is no mixing between the two scalar fields.

The squared masses receive quadratically divergent corrections:

$$
m_{h}^{2} \approx m_{h}^{0^{2}}
$$




\begin{tabular}{|c|c|c|c|c|c|c|}
\hline & $\mathrm{SU}(3)_{C}$ & $\mathrm{SU}(2)_{L}$ & $\mathrm{U}(1)_{Y}$ & $\mathrm{SU}(3)_{\bar{C}}$ & $\mathrm{SU}(2)_{\bar{L}}$ & $\mathrm{U}(1)_{\bar{L}}$ \\
\hline$e_{R}$ & $\mathbf{1}$ & $\mathbf{1}$ & -1 & $\mathbf{1}$ & $\mathbf{1}$ & 0 \\
\hline$L_{L}=\left(\begin{array}{c}\nu_{L} \\
e_{L}\end{array}\right)$ & $\mathbf{1}$ & $\mathbf{2}$ & $-1 / 2$ & $\mathbf{1}$ & $\mathbf{1}$ & 0 \\
\hline$u_{R}$ & $\mathbf{3}$ & $\mathbf{1}$ & $2 / 3$ & $\mathbf{1}$ & $\mathbf{1}$ & 0 \\
\hline$d_{R}$ & $\mathbf{3}$ & $\mathbf{1}$ & $-1 / 3$ & $\mathbf{1}$ & $\mathbf{1}$ & 0 \\
\hline$Q_{L}=\left(\begin{array}{c}u_{L} \\
d_{L}\end{array}\right)$ & $\mathbf{3}$ & $\mathbf{2}$ & $1 / 6$ & $\mathbf{1}$ & $\mathbf{1}$ & 0 \\
\hline$h=\left(\begin{array}{c}h^{+} \\
h^{0}\end{array}\right)$ & $\mathbf{1}$ & $\mathbf{2}$ & $1 / 2$ & $\mathbf{1}$ & $\mathbf{1}$ & 0 \\
\hline \hline$f_{R}^{2}$ & $\mathbf{1}$ & $\mathbf{1}$ & 0 & $\mathbf{1}$ & $\mathbf{1}$ & -1 \\
\hline$F_{L}=\left(\begin{array}{c}f_{L}^{1} \\
f_{L}^{2}\end{array}\right)$ & $\mathbf{1}$ & $\mathbf{1}$ & 0 & $\mathbf{1}$ & $\mathbf{2}$ & $-1 / 2$ \\
\hline$k_{R}^{1}$ & $\mathbf{1}$ & $\mathbf{1}$ & 0 & $\mathbf{3}$ & $\mathbf{1}$ & $2 / 3$ \\
\hline$k_{R}^{2}$ & $\mathbf{1}$ & $\mathbf{1}$ & 0 & $\mathbf{3}$ & $\mathbf{1}$ & $-1 / 3$ \\
\hline$K_{L}=\left(\begin{array}{c}k_{L}^{1} \\
k_{L}^{2}\end{array}\right)$ & $\mathbf{1}$ & $\mathbf{1}$ & 0 & $\mathbf{3}$ & $\mathbf{2}$ & $1 / 6$ \\
\hline$\Phi=\left(\begin{array}{c}\phi^{+} \\
\phi^{0}\end{array}\right)$ & $\mathbf{1}$ & $\mathbf{1}$ & 0 & $\mathbf{1}$ & $\mathbf{2}$ & $1 / 2$ \\
\hline
\end{tabular}

Table 1: Particle content of the minimal SM replica model. 


$$
+\frac{3 g^{2} \Lambda^{2}}{32 \pi^{2} m_{W}^{2}}\left(m_{h}^{2}+2 m_{W}^{2}+m_{Z}^{2}-4 \sum_{f}\left(\frac{n_{f}}{3}\right) m_{f}^{2}\right)+\frac{\Lambda^{2}}{4 \pi^{2}} \alpha,
$$

where $m_{h}$ is the mass of the physical standard model Higgs boson $h$ and

$$
\begin{aligned}
m_{\phi}^{2} \approx & m_{s}^{0^{2}}+\alpha v^{2} \\
& +\frac{3 \Lambda^{2}}{16 \pi^{2}}\left(4 \lambda+\frac{3}{2} g^{2}+\frac{1}{2} g^{\prime 2}-4 \sum_{f c}\left(\frac{n_{f c}}{3}\right) \lambda_{f c}^{2}\right)+\frac{\Lambda^{2}}{4 \pi^{2}} \alpha,
\end{aligned}
$$

where $m_{\phi}$ is the mass of the copy of the standard model Higgs boson and $\lambda_{f c}$ are the Yukawa couplings, note that we have $\lambda_{f c}=\lambda_{f}$. Note that if we assume that the parameters of the second gauge group $\mathrm{G}_{n}$ are identical to those of $\mathrm{G}_{S M}$ besides the sign of the mass of the scalar doublets there is only one relation that needs to be fulfilled:

$$
\alpha=\frac{3 g^{2}}{8 m_{W}^{2}}\left(4 \sum_{f}\left(\frac{n_{f}}{3}\right) m_{f}^{2}-2 m_{W}^{2}-m_{Z}^{2}-m_{h}^{2}\right) \approx 2.13 .
$$

For the numerical estimate we used $m_{h}=130 \mathrm{GeV}$ and took only the top quark into account. It would imply a mass of approximatively $m_{\Phi}=$ $\sqrt{\frac{m_{H}^{2}}{2}+\alpha v^{2}} \approx 270 \mathrm{GeV}$ for the second neutral scalar boson. It is therefore natural to have a cancellation, or near cancellation, of the quadratic divergences if $\alpha$ is of order one. On the other hand, the corrections induced at two loops are not vanishing. This is why we claim that the naturalness problem is under control if there is a fundamental cutoff for the model around $10 \mathrm{TeV}$.

\section{Signatures}

A signature of this class of models is again a decay of the standard model Higgs boson to particles that are not charged under $\mathrm{SU}(2)_{L} \times \mathrm{U}(1)_{Y}$. But, if only the gauge symmetry describing the gauge interactions is broken, the new physics signals are much more subtle. The measurement of the Higgs boson self-coupling (c.f. figure (II) becomes very interesting. In that case a sizable new physics effect is expected. At a linear collider with a center of mass of $1000 \mathrm{GeV}$ one expects $\sigma\left(e^{+}+e^{-} \rightarrow \phi \phi \bar{\nu} \nu\right) \approx 7.4 \times 10^{-6} \mathrm{pb}$. This must be compared to the standard model cross section $\sigma\left(e^{+}+e^{-} \rightarrow h h \bar{\nu} \nu\right)=$ $8.9 \times 10^{-5} \mathrm{pb}$, assuming a Higgs boson mass of $130 \mathrm{GeV}$. We have done these estimates using CompHEP [16], taking only the diagrams corresponding to figure (II) and its standard model counterpart into account. A large effect due 


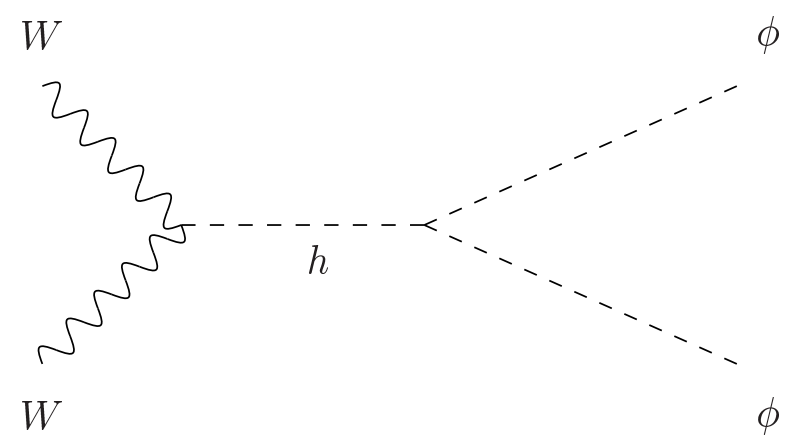

Figure 1: Extraction of the Higgs bosons coupling. The W-bosons are emitted by the colliding fermions.

to the replica sector should be observed. This represents a further motivation for a measurement of the Higgs boson self-coupling at a future linear collider. The signal is a missing energy corresponding to two scalar fields with a mass around $270 \mathrm{GeV}$.

\subsection{A high energy completion?}

The cutoff scale can be shifted to a higher scale if we consider $N$ replicas or copies of the standard model: $\left(\mathrm{SU}(3)_{\bar{C}} \times \mathrm{SU}(2)_{\bar{L}} \times \mathrm{U}(1)_{\bar{Y}}\right)^{N} \times \mathrm{SU}(3)_{C} \times$ $\mathrm{SU}(2)_{L} \times \mathrm{U}(1)_{Y}$. In our case each $\mathrm{SU}(3) \times \mathrm{SU}(2) \times \mathrm{U}(1)$ group has three generations. Note that our model should not be confused with the anti grand unification model proposed in [17], where $N$ stands for the generation number. The vanishing of the quadratic divergences appearing at $N$ loops requires that $N$ equations are fulfilled. Note that if we have $N$ copies of the standard model, we have $N+(N-1)+(N-2)+(N-3)+(N-4)+\ldots=$ $\sum_{K=0}^{N}(N-K)$ parameters $\lambda_{N}$ at our disposition. In that case the $N$ equations can be fulfilled and the scale for the fundamental cutoff can be shifted as high as it is necessary for model building issues. Note that this approach predicts numerous new particles that are stable and very weakly interacting with the usual matter. 


\section{Second case: splitting the scales}

We now come to the second case mentioned previously, namely the case where the scale for new physics is not far away from the cutoff scale. In order to illustrate the idea, we shall consider a generic two Higgs doublets model in the limit where the mass of one of the Higgs doublets $h$ is low lying, whereas the mass of the second Higgs doublet $H$ mass is high. The Veltman's relations for a generic two Higgs doublets model have been considered in [18. The fermions are assumed to couple to both scalar doublets, the hypercharge of $H$ is taken to be equal to that of $h$. This point is however not crucial, the only requirement is that the lightest of the Higgs bosons couples to all fermions in order to reproduce the standard model in the decoupling limit we shall consider. In the sequel we shall assume that the mass of the boson $H$ is rather near to the cutoff scale. For this reason, the possible neutral flavor changing decays are strongly suppressed. Furthermore we assume that most of the symmetry breaking is due to the low lying doublet, i.e. $v_{1} \approx v=174$ $\mathrm{GeV}$ and $v_{2} \approx 0$. In that limit the masses of the charged and CP odd Higgs bosons are of the order of the heavy neutral scalar boson. The assumption $v_{2} \approx 0$ implies that the coefficients of the operators $h^{\dagger} h h^{\dagger} H$ or $H^{\dagger} H H^{\dagger} h$ are nearly vanishing. Again the important terms of the potential for our consideration are:

$$
\alpha h^{\dagger} h H^{\dagger} H+\beta h^{\dagger} H H^{\dagger} h
$$

Under the assumptions mentioned above, the leading radiative corrections to the squared masses of the Higgs bosons are given by:

$$
\begin{aligned}
m_{h}^{2} \approx & m_{h}^{0^{2}} \\
& +\frac{3 g^{2} \Lambda^{2}}{32 \pi^{2} m_{W}^{2}}\left(m_{h}^{2}+2 m_{W}^{2}+m_{Z}^{2}-4 \sum_{f}\left(\frac{n_{f}}{3}\right) m_{f}^{2}\right) \\
& +\frac{1}{4 \pi^{2}}\left(\alpha+\frac{1}{2} \beta\right)\left(\Lambda^{2}-m_{H}^{2} \ln \frac{\Lambda^{2}+m_{H}^{2}}{m_{H}^{2}}\right),
\end{aligned}
$$

and

$$
\begin{aligned}
m_{H}^{2} \approx & m_{H}^{0^{2}}+(\alpha+\beta) v^{2} \\
& \quad+\frac{3 g^{2}}{32 \pi^{2} m_{W}^{2}}\left(4 v^{2} \lambda_{2}+2 m_{W}^{2}+m_{Z}^{2}-4 \sum_{f}\left(\frac{n_{f}}{3}\right) m_{f}^{2}\right) \times
\end{aligned}
$$




$$
\begin{aligned}
& \left(\Lambda^{2}-m_{H}^{2} \ln \frac{\Lambda^{2}+m_{H}^{2}}{m_{H}^{2}}\right) \\
& +\frac{1}{4 \pi^{2}}\left(\alpha+\frac{1}{2} \beta\right)\left(\Lambda^{2}-m_{H}^{2} \ln \frac{\Lambda^{2}+m_{H}^{2}}{m_{H}^{2}}\right),
\end{aligned}
$$

where $\lambda_{2}$ is the self-coupling of the second scalar doublet $H$. Now we can require the cancellation, or near cancellation, of the quadratic corrections to the squared mass of $h$. One obtains:

$$
\alpha+\frac{1}{2} \beta \approx 3 \frac{3 g^{2}}{8 m_{W}^{2}}\left(4 \sum_{f}\left(\frac{n_{f}}{3}\right) m_{f}^{2}-m_{h}^{2}-2 m_{W}^{2}-m_{Z}^{2}\right) \approx 6.4
$$

using again $m_{h}=130 \mathrm{GeV}$ for the numerical estimate. The first factor 3 will be explained bellow. Note that although we could adjust $\alpha$ and $\beta$ to cancel the quadratically divergent corrections to the mass of the Higgs boson $h$, the corrections to the second Higgs boson mass are in general rather large. This is the reason why we assume that the mass of the second Higgs boson $m_{H}$ is not much lower than the cutoff, in which case the quadratic corrections to $m_{H}^{2}$ are small compared to its bare value. The mass of the second scalar field $H$ could be around $9 \mathrm{TeV}$ if we take a cutoff of $10 \mathrm{TeV}$. But, if the mass of the scalar field is not much below the cutoff it is important to consider the full one loop corrections. This explains the term $\left(\Lambda^{2}-m_{H}^{2} \ln \frac{\Lambda^{2}+m_{H}^{2}}{m_{H}^{2}}\right)$ in eq. (18). For $m_{H}=9 \mathrm{TeV}$ and $\Lambda=10 \mathrm{TeV}$, one finds that $\Lambda^{2}$ should be replace by approximatively $\Lambda^{2} / 3$. This explains the first factor 3 in eq. (19).

Note that in that case, it seems very difficult to push the cutoff scale upwards in a natural way. It has recently been pointed out that the Higgs mechanism can be induced by a large splitting between the two masses of a two Higgs doublets model [19]. It is thus possible to construct a two Higgs doublets model that is natural up to a scale of $10 \mathrm{TeV}$ which furthermore triggers the Higgs mechanism. It will be very difficult to differentiate this model from the standard model since the low energy theory below the $9 \mathrm{TeV}$ scale is just the standard model.

We finally want to point out that it could be possible to shift the supersymmetry breaking scale from one $\mathrm{TeV}$ to about $10 \mathrm{TeV}$ if new operators are added, for example, to the minimal supersymmetric model. In that case one would have to assume that the logarithmic terms that lead to a new naturalness problem if the supersymmetric scale is higher than one $\mathrm{TeV}$ are 
cancelled by these new operators. We note that supersymmetry would provide an ultraviolet completion to the model.

\section{Conclusions}

We have described a class of models that are semi-natural in the sense proposed by Veltman in 4 a long time ago. The idea proposed by Veltman considered in a modern framework provides an interesting alternative to the Little Higgs models whose minimal versions are potentially severely constrained by experiments. In our approach a cancellation of the radiative corrections are only semi-natural because there is no symmetry that imposes them. Nevertheless this is a possibility that cannot be ignored. The new term allowing the cancellation can be generated by different types of models. We have described two classes of models where such a term appears.

The first type of models is a direct product of the standard model and of its replica. If both $\mathrm{SU}(2) \times \mathrm{U}(1)$ symmetries are broken, the Higgs physics is considerably affected. If only the electroweak gauge symmetry is broken, then the new physics effects are much more subtle and only a measurement of the scalar potential will allow to distinguish our scenario from the standard model. This class of model is particularly appealing since the cutoff scale can, in principal, be shifted to any desirable scale by introducing more replicas of the standard model.

We then described a two Higgs doublets model where a cancellation of the one loop quadratic divergences is possible. The second class of model will be much more difficult to distinguish from the standard model and finding a deviation will require to test the very high energy region around the mass of the second Higgs boson which can be as high as $9 \mathrm{TeV}$.

We would like to emphasize the main point of this paper is that due to an "accidental" cancellation of the one loop quadratic divergences, the true scale for the naturalness problem might be around $10 \mathrm{TeV}$ rather than around $1 \mathrm{TeV}$ as it is usually argued. We do not claim, as Veltman did, that a formula such as eq. (11) could be used to compute the Higgs boson's mass. We propose to use Veltman's relation as a criterion for the naturalness of the model. One could imagine introducing different cutoffs for the different particles entering the loop, but even in that case there will be a Veltman type formula that is either fulfilled or not. If it is fulfilled or even approximatively

fulfilled, it can be interpreted as a sign that the true scale for the naturalness 
problem is $10 \mathrm{TeV}$ rather than $1 \mathrm{TeV}$.

Ultraviolet completions of the models we are proposing have not been considered. One could imagine having $N$ copies of the standard model in the case where the mass scale of both scalars is much below the cutoff scale. Note that if $N \geq 14$, one could consider a cutoff of the order of the grand unification scale. One could also imagine a supersymmetric high energy completion in the case where the mass of one of the scalar doublets is just below the cutoff scale. Another well studied possibility is that the fundamental scale of physics is in the $\mathrm{TeV}$ region if nature has more than four dimensions [9]. In our approach the scale for these new extra-dimensions might be around $10 \mathrm{TeV}$ and still provide a solution to the naturalness problem.

\section{Acknowledgments}

The author is grateful to M. Graesser and S. Su for enlightening discussions. Furthermore he would like to thank M. Schmaltz for a useful and interesting communication and P. Bambade for triggering his interest in the question of the measurement of the Higgs boson self-coupling.

\section{References}

[1] S. L. Glashow, Nucl. Phys. 22, 579 (1961); S. Weinberg, Phys. Rev. Lett. 19, 1264 (1967); A. Salam, Elementary Particle Physics, in Proceedings of the 8th Nobel Symposium, 1968.

[2] H. Fritzsch and M. Gell-Mann, "Current algebra: Quarks and what else?," Published in Physics, Proceedings of the XVI International Conference on High Chicago 1972 p.135 (J. D. Jackson, A. Roberts, eds.), arXiv:hep-ph/0208010, H. Fritzsch, M. Gell-Mann and H. Leutwyler, Phys. Lett. B 47, 365 (1973); H. Fritzsch and M. Gell-Mann, "Light Cone Current Algebra," arXiv:hep-ph/0301127.

[3] P. W. Higgs, Phys. Lett. 12 (1964) 132; Phys. Rev. Lett. 13 (1964) 508; Phys. Rev. 145 (1966) 1156; F. Englert and R. Brout, Phys. Rev. Lett. 13321 (1964); G. S. Guralnik, C. R. Hagen and T. W. Kibble, Phys. Rev. Lett. 13585 (1964); T. W. Kibble; Phys. Rev. 155, 1554 (1967).

[4] M. Veltman, Acta Phys. Polon. B 12, 437 (1981). 
[5] G. 't Hooft, in "Recent Developments In Gauge Theories", Cargesè 1979, ed. G. 't Hooft et al. Plenum Press, New York, 1980, Lecture III, p.135; L. Susskind, Phys. Rev. D 20, 2619 (1979).

[6] G. 't Hooft, Nucl. Phys. B 35, 167 (1971); Nucl. Phys. B 33, 173 (1971); G. 't Hooft and M. J. Veltman, Nucl. Phys. B 44, 189 (1972); Nucl. Phys. B 50, 318 (1972).

[7] C. T. Hill and E. H. Simmons, arXiv:hep-ph/0203079.

[8] H. E. Haber and G. L. Kane, Phys. Rept. 117, 75 (1985).

[9] N. Arkani-Hamed, S. Dimopoulos and G. R. Dvali, Phys. Lett. B 429, 263 (1998) arXiv:hep-ph/9803315; L. Randall and R. Sundrum, Nucl. Phys. B 557, 79 (1999) arXiv:hep-th/9810155; L. Randall and R. Sundrum, Phys. Rev. Lett. 83, 3370 (1999) arXiv:hep-ph/9905221.

[10] H. Georgi and A. Pais, Phys. Rev. D 12, 508 (1975); D. B. Kaplan and H. Georgi, Phys. Lett. B 136, 183 (1984).

[11] N. Arkani-Hamed, A. G. Cohen, E. Katz, A. E. Nelson, T. Gregoire and J. G. Wacker, JHEP 0208, 021 (2002) arXiv:hep-ph/0206020; N. Arkani-Hamed, A. G. Cohen, E. Katz and A. E. Nelson, JHEP 0207, 034 (2002) arXiv:hep-ph/0206021.

[12] C. Csaki, J. Hubisz, G. D. Kribs, P. Meade and J. Terning, arXiv:hep-ph/0211124 J. L. Hewett, F. J. Petriello and T. G. Rizzo, arXiv:hep-ph/0211218 G. Burdman, M. Perelstein and A. Pierce, arXiv:hep-ph/0212228, T. Han, H. E. Logan, B. McElrath and L. T. Wang, arXiv:hep-ph/0301040.

[13] R. Decker and J. Pestieau, Mod. Phys. Lett. A 4, 2733 (1989); E. Ma, Phys. Rev. D 47, 2143 (1993) arXiv:hep-ph/9209221.

[14] C. F. Kolda and H. Murayama, JHEP 0007, 035 (2000) arXiv:hep-ph/0003170.

[15] K. Hagiwara et al. [Particle Data Group Collaboration], Phys. Rev. D 66, 010001 (2002). 
[16] A. Pukhov et al., "CompHEP: A package for evaluation of Feynman diagrams and integration over multi-particle phase space. User's manual for version 33," hep-ph/9908288.

[17] D. L. Bennett, H. B. Nielsen and I. Picek, Phys. Lett. B 208, 275 (1988).

[18] C. Newton and T. T. Wu, Z. Phys. C 62, 253 (1994); see also A. A. Andrianov, R. Rodenberg and N. V. Romanenko, Nuovo Cim. A 108, 577 (1995) arXiv:hep-ph/9408301; E. Ma, Int. J. Mod. Phys. A 16, 3099 (2001) arXiv:hep-ph/0101355.

[19] X. Calmet, arXiv:hep-ph/0206091. 